\title{
On the Oppenheimer-Volkoff Equations in General Relativity
}

\author{
Joel Smoller \& Blake Temple \\ Communicated by C. DAFERMOS
}

\begin{abstract}
We introduce a new formulation of the Oppenheimer-Volkoff $(\mathrm{O}-\mathrm{V})$ equations, a system of ordinary differential equations that models the interior of a star in general relativity, and we use this to give a completely rigorous mathematical analysis of solutions. In particular, we prove that, under mild assumptions on the equation of state, black holes never form in solutions of the O-V equations. As a corollary, this implies that the portion of the empty-space Schwarzschild solution inside the Schwarzschild radius cannot be obtained as a limit of $\mathrm{O}-\mathrm{V}$ solutions having non-zero density. We also prove that if the density $\rho$ at radius $r$ is ever larger than $\frac{3}{4 \pi} \frac{M(r)}{r^{3}}$, where $M(r)$ is the total mass inside radius $r$, then $M$ must become negative for some positive radius. We interpret $M<0$ as a condition for instability because we show that if the pressure is a decreasing function of $r$, then $M(r)<0$ at some $r>0$ implies that the pressure tends to infinity before $r=0$.
\end{abstract}

\section{Introduction}

The Oppenheimer-Volkoff (O-V) equations describe the pressure gradient inside a static fluid sphere when the gravitational forces are modeled by Einstein's Theory of General Relativity, and thus they provide a model for the interior of a star [1]. The O-V equations form a system of two non-autonomous, nonlinear, ordinary differential equations in the unknown pressure $p$, the density $\rho\left(\rho c^{2}\right.$ is the massenergy density, $c$ is the speed of light), and the "mass function" $M$, where $M \equiv M(r)$ denotes the total mass inside radius $r$. Although an equation of state of the form $p=p(\rho)$ closes the $\mathrm{O}-\mathrm{V}$ system, the $\mathrm{O}-\mathrm{V}$ system applies to equations of state which can allow, e.g., for temperature dependence.

In this paper we introduce a new formulation of the $\mathrm{O}-\mathrm{V}$ system, and use this to rigorously describe the behavior of solutions in the large, assuming only mild restrictions on the equation of state (namely, (2.10) and (2.11) below.) Specifically, 
we describe the behavior of solutions for values of the radius $r<r_{0}$, starting from initial data given at $r=r_{0}>0$, where $r_{0}$ could, for example, be taken to be the outer surface of a star. This differs from the standard approach which pose initial conditions at the center $r=0$. Our equations are written in terms of a new variable $z \equiv \frac{\rho}{\bar{\rho}}$ which we introduce, where $\bar{\rho}(r) \equiv \frac{3}{4 \pi} \frac{M(r)}{r^{3}}$ denotes the average density inside radius $r$. The variable $z$ is quite natural for this problem. In particular, we show below that $z \rightarrow+\infty$ as $r \rightarrow r_{1}+$ whenever there is a positive radius $r_{1}>0$ at which $M\left(r_{1}\right)=0$. Thus, if one restricts attention to the class of solutions of the $\mathrm{O}-\mathrm{V}$ equations satisfying $M \geqq 0$ (these are usually considered to be the only physically relevant solutions [1, page 300]), then $r_{1}$ marks the inner boundary of the "physical"solution. Indeed, we show that if $M(r)<0$ for some $r>0$, then either $p^{\prime}<0$ cannot hold for all $r<r_{1}$, or else $p$ tends to infinity before $r=0$. We interpret this as a condition for instability.

The occurrence of $M\left(r_{1}\right)=0$ happens when there is no additional mass available to continue a positive mass solution of the O-V equations inward beyond $r=r_{1}$. When $M\left(r_{1}\right)=0$ for $r_{1}>0$, we prove that the $\mathrm{O}-\mathrm{V}$ solution can be continued to values $r<r_{1}, r$ near $r_{1}$, but for these values, $M<0$. Thus, $\rho$ and $p$ must have finite non-zero values at $r=r_{1}$ even though $M\left(r_{1}\right)=0$ marks the end of the "physical regime" in the above sense. On the other hand, the variable $z$ tends to infinity at the inner boundary $r_{1}$ of the solution, because $z=\frac{\rho}{\bar{\rho}}$ and $\bar{\rho} \rightarrow 0$ at $r=r_{1}$. In this sense the variable $z$ is more natural than $\rho$ or $p$ for this problem.

In this paper we also prove that for solutions of the O-V system, if the density ever exceeds the average density, then $M=0$ at some $r_{1}>0$. (This is not predicated on the a priori assumption that black holes never form in solutions.) Since $M^{\prime}(r)>$ 0 , this provides the following a priori bound on the density profile of a static fluid sphere having positive mass function $M(r) \geqq 0$ in general relativity. (The only restrictions on the equation of state are imposed by (2.10) and (2.11) below.)

Theorem 1. If a static fluid sphere satisfies $M(r) \geqq 0$ for $r \geqq r_{1} \geqq 0$, then for every $r_{0}>r_{1}$, the density profile $\rho(r)$ satisfies

$$
\rho(r) \leqq \frac{3}{4 \pi} \frac{M(r)}{r^{3}} \leqq \frac{3}{4 \pi} \frac{M\left(r_{0}\right)}{r^{3}}
$$

for all $r_{1} \leqq r \leqq r_{0}$.

In particular, (1.1) implies that if $\rho(r)>\frac{3}{4 \pi} \frac{M(r)}{r^{3}}$ at any radius $r$, then $M(r)<0$ at some positive radius $r$. One can interpret this as a local condition for instability which is independent of the equation of state.

We also prove that black holes cannot form in solutions of the $\mathrm{O}-\mathrm{V}$ equations when the pressure is non-zero (assuming only (2.10) and (2.11) below). That is, when the pressure is non-zero, black holes cannot form in static, spherically symmetric solutions of the Einstein equations for a perfect fluid. This implies that the portion of the empty-space Schwarzschild solution inside the Schwarzschild radius is "disconnected" from the rest of the solution space of the O-V system in the sense that it cannot be obtained as a limit of $\mathrm{O}-\mathrm{V}$ solutions having non-zero density. 


\section{Statement of Results}

We now give a precise statement of our main results. The $\mathrm{O}-\mathrm{V}$ system is given by (cf. [1])

$$
\begin{gathered}
-r^{2} \frac{d p}{d r}=\mathscr{G} M \rho\left(1+\frac{p}{\rho c^{2}}\right)\left(1+\frac{4 \pi r^{3} p}{M c^{2}}\right) A^{-1} \\
\frac{d M}{d r}=4 \pi \rho r^{2} .
\end{gathered}
$$

This is a system of two ordinary differential equations in the unknown functions $p=p(r), \rho=\rho(r)$, and $M=M(r)$, where $p$ denotes the pressure, $\rho c^{2}$ denotes the the mass-energy density, $c$ denotes the speed of light, $M(r)$ denotes the total mass inside radius $r, \mathscr{G}$ denotes Newton's gravitational constant, and

$$
A=A(r) \equiv 1-2 \frac{\mathscr{G}}{c^{2}} \frac{M(r)}{r} .
$$

WeINBERG [1, page 301], refers to the Oppenheimer-Volkoff equation (2.1) as the fundamental equation of Newtonian astrophysics, with general-relativistic corrections supplied by the last three factors.

The constant $\mathscr{G}$ enters through the Einstein equations

$$
G=\frac{8 \pi \mathscr{G}}{c^{4}} \mathscr{T}
$$

where $G$ is the Einstein tensor and $\mathscr{T}$ denotes the stress-energy tensor. Solutions of (2.1) and (2.2) determine a Lorentzian metric tensor $g$ of the form

$$
d s^{2}=-B(r) d(c t)^{2}+A(r)^{-1} d r^{2}+r^{2}\left(d \theta^{2}+\sin ^{2}(\theta) d \phi^{2}\right)
$$

that satisfies the Einstein equations (2.4) when $\mathscr{T}$ is the stress-energy tensor for a perfect fluid:

$$
\mathscr{T}_{i j}=\left(p+\rho c^{2}\right) u_{i} u_{j}+p g_{i j} .
$$

For the metric (2.5), the function $A(r)$ is defined by (2.3), and the function $B \equiv B(r)$ is determined by the equation

$$
\frac{B^{\prime}}{B}=-2 \frac{p^{\prime}}{p+\rho c^{2}} .
$$

The metric (2.5) is spherically symmetric, time-independent, and the fluid 4velocity is given by $u_{0}=\sqrt{B}$ and $u_{r}=u_{\theta}=u_{\phi}=0$ [1], so that the fluid is fixed in the $(t, r, \theta, \phi)$-coordinate system.

To close the $\mathrm{O}-\mathrm{V}$ system $(2.1),(2.2)$, one usually assigns an equation of state that relates $p$ and $\rho$, and then $B(r)$ is determined by (2.7). However, to be more general (for example, to allow for temperature dependence), we assume only that

$$
\mu=\frac{p}{\rho},
$$




$$
\sigma=\frac{d p / d r}{d \rho / d r}
$$

satisfy the a priori bounds

$$
\begin{gathered}
0 \leqq \tilde{\mu}<\Sigma<\infty, \\
0<\Sigma_{1}<\tilde{\sigma}<\Sigma_{2}<\infty,
\end{gathered}
$$

where throughout this paper we use $\tilde{\mu}$ and $\tilde{\sigma}$ to denote the dimensionless quantities

$$
\begin{gathered}
\tilde{\mu}=\frac{\mu}{c^{2}}, \\
\tilde{\sigma}=\frac{\sigma}{c^{2}} .
\end{gathered}
$$

That is, our results apply to any solution for which (2.10) and (2.11) hold. In particular, our results apply to any barytropic equation of state in which $\mu(\rho) \equiv$ $\frac{p}{\rho}<\Sigma$ and $0<\Sigma_{1}<\sigma(\rho) \equiv \frac{d p}{d \rho}<\Sigma_{2}$. In this case, for physically reasonable equations of state, $\sigma$ is the square of the sound speed and must be bounded by $c^{2}$. Thus, in the case of a barytropic equation of state $p=p(\rho)$, it is reasonable to assume that the equation of state satisfies the bounds (2.10), (2.11).

However, our results apply even when the pressure $p$ is not a given function of the density $\rho$ alone. For example, when there is a more general equation of state allowing for temperature dependence in the problem, our results still apply to any solution so long as the bounds (2.10) and (2.11) hold. To motivate (2.10) and (2.11), we note that these bounds have a very natural interpretation in the case of an ideal gas in the nonrelativistic limit. Here the ideal gas law reads

$$
p=\mathscr{R} T \rho,
$$

where $\mathscr{R}$ is a constant and $T$ denotes the temperature. In this case, the sound speed $\sqrt{\sigma_{s}}$ is given by

$$
\sqrt{\sigma_{s}}=\sqrt{\gamma(T) \cdot \mathscr{R} T}
$$

where $\gamma(T)$ is the ratio of specific heats, a constant in the case of a polytropic equation of state [2]. Thus when (2.14) holds,

$$
\sigma=\frac{p^{\prime}}{\rho^{\prime}}=\frac{\mathscr{R} T^{\prime} \rho}{\rho^{\prime}}+\mathscr{R} T
$$

where the prime denotes differentiation with respect to $r$ in a given solution. Now the second term on the right-hand side of (2.15) is the square of the sound speed divided by $\gamma(T)>0$, and the first term on the right-hand side of $(2.15)$ is positive if $T^{\prime}<0$ and $\rho^{\prime}<0$, both reasonable assumptions. (In this case the first term on the right-hand side of (2.15) is a measure of the temperature gradient inside the star.) We conclude that in the case of an ideal gas, the bound $0<\Sigma_{1}<\tilde{\sigma}$ holds when there is a negative temperature gradient. Note also that in light of the $\mathrm{O}-\mathrm{V}$ equation, assuming (2.10) and (2.11) is equivalent to restricting the class of solutions to those for which $-\infty<\rho^{\prime}<0$ when $\rho, M, p$ are positive and finite, and $A>0$. In 
summary, our results apply to equations of state with temperature dependence so long as the bounds (2.10) and (2.11) hold.

In order to obtain estimates on any O-V solution for which (2.10) and (2.11) hold, we proceed as follows. Namely, we take $\mu(r)$ and $\sigma(r)$ to be any given known functions defined on $\left[0, r_{0}\right]$ that are smooth and satisfy these bounds. We then use the expressions $\frac{p}{\rho}=\mu$ and $\frac{p^{\prime}}{\rho^{\prime}}=\sigma$ to close the $\mathrm{O}-\mathrm{V}$ equations (2.1), (2.2) in the variables $\rho$ and $M$ by writing (2.1) as

$$
-r^{2} \frac{d \rho}{d r}=\frac{\mathscr{F}}{\sigma} M \rho(1+\tilde{\mu})\left(1+\frac{4 \pi r^{3} \tilde{\mu} \rho}{M}\right) A^{-1} .
$$

We now take (2.16) and (2.2) as a system of two non-autonomous ordinary differential equations in the two unknowns $\rho$ and $M$. It is important to note that for fixed functions $\mu(r)$ and $\sigma(r)$, the solutions $(\rho(r), M(r))$ that satisfy (2.16) and (2.2) do not in general satisfy (2.10) and (2.11). However, because $\mu$ and $\sigma$ are arbitrary, we can conclude that any actual solution of the original O-V system such that the bounds (2.10) and (2.11) hold, must satisfy the conclusions of our theorem. Note finally that in the case when we are given an equation of state of the form $p=p(\rho)$, the system (2.16), (2.2) is exactly equivalent to the original O-V system (2.1), (2.2).

To study solutions of the O-V system (2.16), (2.2) for fixed $\mu(r)$ and $\sigma(r)$, we introduce the variables $\bar{\rho}$ and $z$ by

$$
\begin{gathered}
\bar{\rho}=\frac{3}{4 \pi} \frac{M}{r^{3}}, \\
z=\frac{\rho}{\bar{\rho}} .
\end{gathered}
$$

The variables $\bar{\rho}(r)$ and $z$ arise naturally in our previous work on constructing exact shock-wave solutions of the Einstein equations $[3,4,5]$. The variable $\bar{\rho}(r)$ is the average density of a solution inside radius $r$, and in [4], $\bar{\rho}$ is also the density of the inner Friedmann-Robertson-Walker metric that matches the (outer) O-V solution Lipschitz continuously across a shock interface positioned at $r$. The variable $z$ measures the strength of the density jump across the shock, and the shock wave is an outgoing shock modeling an explosion in the case when $0<z<1$ [5]. Although these variables arise in the shock-wave construction problem, they are defined solely in terms of the O-V solution.

In order to study solutions of the O-V system, we transform (2.1), (2.2) into an equivalent system in the variables $z$ and $A$. To this end, multiply $M$ into the second of the last three factors in (2.1), use the identities (2.17), (2.18), and (2.3) together with $p^{\prime}=\sigma \rho^{\prime}$ and $p=\mu \rho$ to eliminate $p, \rho$ and $M$ in favor of $z$ and $A$ in (2.1), (2.2). This gives the equivalent system

$$
\begin{aligned}
& \frac{d z}{d r}=-C \frac{z}{A}\left(\frac{1-A}{r}\right), \\
& \frac{d A}{d r}=(1-3 z)\left(\frac{1-A}{r}\right),
\end{aligned}
$$


where

$$
C \equiv \frac{\left(1+\frac{\mu}{c^{2}}\right)\left(1+\frac{3 \mu z}{c^{2}}\right)}{2 \frac{\sigma}{c^{2}}}-\frac{3(1-z) A}{(1-A)} .
$$

In terms of $z$ and $A$, equation (2.7) becomes

$$
\frac{B^{\prime}}{B}=\frac{c^{2}}{r}\left(1+3 \frac{\mu z}{c^{2}}\right)\left(\frac{1-A}{A}\right) .
$$

The main advantage of the formulation (2.19), (2.20) is that all of the dependence on $\mu$ and $\sigma$ (i.e., on the equation of state) is isolated in the factor $C$, and when $z>1$, $C$ is positive, and can be estimated from above and below. Moreover, we will see that we can use equations (2.19) and (2.20) to estimate $A$ in terms of $z$ by substituting into one of the equations the expression for $\left(\frac{1-A}{r}\right)$ obtained from the other, thereby obtaining an equation which is independent of $r$. However, the main difficulty in analyzing solutions of system (2.19), (2.20) lies in the fact that the function $C$ in (2.21) need not be positive when $z<1$. Indeed, for the exact solution of the $\mathrm{O}-\mathrm{V}$ system studied in [4], i.e, the solution that describes a static, singular, isothermal sphere in general relativity, it is easy to verify that $C$ is identically zero. In this example, $p=\mu \rho$ for $\mu \equiv$ const., $A \equiv$ const., $z \equiv \frac{1}{3}$, and the density profile varies as the inverse square in $r$. Note, finally, that when we assume that $p$ is a given function of $\rho$, the substitution $s=\ln (r)$ reduces the system (2.19), (2.20) to an autonomous system, but since we are interested in quantitative rather than qualitative information about solutions, this gives us no essential simplification.

The main results of this paper are summarized in the following theorem:

Theorem 2. Let $(z(r), A(r))$ denote the smooth (i.e., $\left.C^{1}\right)$ solution of (2.19), (2.20), defined on a maximal interval $\left(r_{1}, r_{0}\right], 0 \leqq r_{1}<r_{0}<\infty$, satisfying the initial conditions $z\left(r_{0}\right)=z_{0}, A\left(r_{0}\right)=A_{0}$, where

$$
0<z_{0}<\infty, \quad 0<A_{0}<1 .
$$

Assume that (2.10) and (2.11) hold. Then $(z(r), A(r))$ satisfies the following inequalities for all $r \in\left(r_{1}, r_{0}\right]$ :

$$
\begin{gathered}
0<z(r)<\infty, \\
0<A(r)<1, \\
B(r)>0, \\
0<M(r)<M\left(r_{0}\right), \quad M^{\prime}(r)>0, \\
\lim _{r \rightarrow r_{1}+} M(r)=0 .
\end{gathered}
$$

Moreover, if $r_{1}>0$, then

$$
\begin{gathered}
\lim _{r \rightarrow r_{1}+} z(r)=+\infty, \\
\lim _{r \rightarrow r_{1}+} A(r)=1,
\end{gathered}
$$




$$
\lim _{r \rightarrow r_{1}+} B(r)=B\left(r_{1}\right)>0
$$

If $r_{1}=0$, then

$$
0 \leqq z(r) \leqq 1
$$

for all $r \in\left(0, r_{0}\right]$, and if $\rho(r)$ has a finite limit at $r_{1}=0$, then (2.30) and (2.31) also hold.

The original variables $\rho$ and $p$ of the $O-V$ system (2.1), (2.2) satisfy the inequalities

$$
\begin{array}{ll}
0<\rho\left(r_{0}\right)<\rho(r)<\rho\left(r_{1}\right)<\infty, & \rho^{\prime}(r)<0, \\
0<p\left(r_{0}\right)<p(r)<p\left(r_{1}\right)<\infty, & p^{\prime}(r)<0 .
\end{array}
$$

Finally, if $r_{1}>0$ and $p^{\prime}<0$ for all $r \leqq r_{1}$, then there exists an $r_{2}, 0 \leqq r_{2}<r_{1}$, such that

$$
\lim _{r \rightarrow r_{2}+} p(r)=+\infty
$$

We remark that (2.28) and (2.29) show that $z$ can only tend to infinity at a positive value of $r$ where $M=0$. Furthermore, it follows that when $r_{1}>0$, the values of $\rho$ and $p$ are bounded on the closed interval $r_{1} \leqq r \leqq r_{0}$. Thus, solutions of the $\mathrm{O}-\mathrm{V}$ system (2.1), (2.2), exist on a larger interval containing $\left[r_{1}, r_{0}\right]$, and for $r<r_{1}, M(r)<0$. Thus (2.19), (2.20) is a more natural formulation in the sense that the breakdown of smooth solutions occurs exactly at $r_{1}$, the point where $M=0$. That is, (2.33) and (2.34) state that $\rho$ and $p$ are finite at $r=r_{1}$ when $r_{1}>0$. This is because the $\mathrm{O}-\mathrm{V}$ equation implies that if $\rho \rightarrow \infty$ at $r=r_{1}$, then $\rho$ must have a non-integrable singularity at $r_{1}$ (this follows by an argument analogous to that following (3.14) below), and thus since

$$
M(r)=M\left(r_{0}\right)-\int_{r}^{r_{0}} 4 \pi \rho(s) s^{2} d s,
$$

$M$ must tend to $-\infty$ if $\rho \rightarrow+\infty$ at $r=r_{1}>0$, thereby violating (2.28). In contrast, if $r_{1}=0$, we see by example that $\rho$ can tend to infinity at $r_{1}$ with $z$ staying bounded. Indeed, this occurs in the case of an isothermal sphere when the equation of state is of the form $p=\sigma \rho, \sigma$ constant; cf. [4].

It is interesting to note that (2.32) implies that if initially $z\left(r_{0}\right)>1$, then blow-up in $z$ must occur before $r=0$, so that $r_{1}>0$. As a corollary, we have the following local condition which implies instability for solutions of the Oppenheimer-Volkoff equations, and (since $M^{\prime}>0$ ) this implies Theorem 1 of the introduction:

Corollary 1. Let $(z(r), A(r))$ be any $C^{1}$ solution of (2.19), (2.20), starting from the initial conditions $z\left(r_{0}\right)=z_{0}, A\left(r_{0}\right)=A_{0}$, satisfying

$$
0<z_{0}<\infty, \quad 0<A_{0}<1,
$$

and assume that (2.10) and (2.11) hold. Then if $z\left(r_{0}\right)>1$, i.e., if

$$
\rho\left(r_{0}\right)>\frac{3}{4 \pi} \frac{M\left(r_{0}\right)}{r_{0}^{3}},
$$

it follows that there exists an $r_{1}, 0<r_{1}<r_{0}$, such that $M\left(r_{1}\right)=0$. 
This result shows that if the equation of state satisfies (2.10) and (2.11), and if (2.37) holds, i.e., if the density of a star at radius $r_{0}$ is too large relative to the total mass $M_{0}$ at $r_{0}$, then no finite pressure at the center of the star is sufficient to "hold the static configuration up", because (2.35) implies that $p$ is unbounded in $r<r_{1}$.

That $A>0$ in solutions of (2.1), (2.2) implies that the portion of the empty-space Schwarzschild solution inside the Schwarzschild radius is "disconnected" from the rest of the solution space of the $\mathrm{O}-\mathrm{V}$ system in the sense that it cannot be obtained as a limit of $\mathrm{O}-\mathrm{V}$ solutions having non-zero density. Indeed, by continuous dependence for ordinary differential equations, it follows that if $A\left(r_{0}\right)=A_{0}$ is fixed and we let $\rho\left(r_{0}\right) \rightarrow 0$, the solution must tend to the empty-space Schwarzschild solution outside the Schwarzschild radius (a solution of (2.1), (2.2)). But since $A \neq 0$ in a solution starting from $\rho\left(r_{0}\right) \neq 0$, and $A<0$ inside the Schwarzschild radius of the Schwarzschild solution, it follows that $\mathrm{O}-\mathrm{V}$ solutions with $\rho\left(r_{0}\right) \neq 0$ diverge from the empty-space Schwarzschild solution near the Schwarzschild radius, and there is no convergence inside the Schwarzschild radius in the limit $\rho\left(r_{0}\right) \rightarrow 0$.

\section{Regularity of the OT Solutions}

In this section we give the proof of Theorem 1 . To start, let $I \equiv\left(r_{1}, r_{0}\right], r_{1} \geqq 0$, be the maximal interval on which $z(r)$ and $A(r)$ are smooth $\left(C^{1}\right)$ solutions of (2.19), (2.20), where $C$ is given by (2.21) and the initial data satisfy (2.23). We begin with the following lemma:

Lemma 1. The metric component A satisfies $A(r)<1$ for all $r \in I$. Moreover, if $r_{1}>0$ and $\lim _{r \rightarrow r_{1}} A(r)=1$, then $\lim _{r \rightarrow r_{1}}+z(r)=+\infty$.

Proof. Assume for contradiction that $A(s)=1$ for some $s \in\left(r_{1}, r_{0}\right)$. Then from (2.20), we have

$$
\frac{d A}{1-A}=(1-3 z) \frac{d r}{r}
$$

and integrating from $s$ to $r_{0}$ gives

$$
\frac{1-A(s)}{1-A_{0}}=\exp \left[-\int_{s}^{r_{0}}(1-3 z) \frac{d r}{r}\right] .
$$

Hence, if $A(s)=1$, it follows that

$$
\int_{s}^{r_{0}}(1-3 z) \frac{d r}{r}=-\infty,
$$

because $s>0$. This is a contradiction since $z(r)$ is assumed to be a smooth function on $\left(r_{1}, r_{0}\right]$; this proves that $A(r)<1$ on $I$.

Now assume that $r_{1}>0$ and $\lim _{r \rightarrow r_{1}} A(r)=1$. Then replacing $s$ by $r_{1}>0$ in (3.1), we see that $\lim _{r \rightarrow r_{1}} z(r)=+\infty$. This completes the proof of Lemma 1 .

Next we prove that if $A$ is positive on a sub-interval $\left(\tilde{r}, r_{0}\right]$ of $I$, then $z(r)$ is positive on the same sub-interval. 
Lemma 2. Assume that $A(r)>0$ on an interval $\left(\tilde{r}, r_{0}\right]$, where $r_{1} \leqq \tilde{r}<r_{0}$. Then $z(r)>0$ for all $r \in\left(\tilde{r}, r_{0}\right]$.

Proof. Let $s \in\left(\tilde{r}, r_{0}\right]$. Then writing (2.20) in the form

$$
\frac{(1-A)}{r}=\frac{A^{\prime}}{1-3 z},
$$

and substituting into (2.19), we have

$$
z^{\prime}=-C \frac{z}{A} \frac{A^{\prime}}{(1-3 z)},
$$

or

$$
\frac{1-3 z}{z} d z=-C \frac{d A}{A}
$$

which gives

$$
\int_{z_{0}}^{z(s)}\left(\frac{1}{z}-3\right) d z=\int_{A(s)}^{A_{0}} C \frac{d A}{A}
$$

Integrating yields

$$
\frac{z(s)}{z_{0}} \exp \left[-3\left(z(s)-z_{0}\right)\right]=\exp \left[\int_{A(s)}^{A_{0}} C \frac{d A}{A}\right] .
$$

But $C$ is continuous on $\left[s, r_{0}\right]$, because $z(r)$ and $A(r)$ are continuous on $\left[\tilde{r}, r_{0}\right]$, and $0<A<1$ on $\left(\tilde{r}, r_{0}\right]$ implies that $A$ is also bounded away from 0 and 1 on the closed sub-interval $\left[s, r_{0}\right] \in I$ (because $I$ is the maximal interval on which $A$ and $z$ are continuous). Thus $C$ is continuous and hence bounded on $\left[s, r_{0}\right]$, so it follows from (3.2) that $z(s)>0$, for all $s \in\left(\tilde{r}, r_{0}\right]$. This proves Lemma 2.

We show that Lemmas 1 and 2 imply that if $r_{1}>0$, then $A>0$ on the entire interval $I$ :

Lemma 3. Assume that $r_{1}>0$. Then $A(r)>0$ on the maximal interval $I=$ $\left(r_{1}, r_{0}\right]$.

Proof. Let $J \equiv\left(\tilde{r}, r_{0}\right] \subset I$ now denote the maximal sub-interval of $I$ on which $A>0$. Then since $I$ is the maximal interval on which $z(r)$ and $A(r)$ are $C^{1}$ functions, it follows that either $\tilde{r}=r_{1}$ (in which case we are done) or else $\tilde{r}>r_{1}$ and $A(\tilde{r})=0$. If $\tilde{r}>r_{1}$ and $A(\tilde{r})=0$, we claim that

$$
\lim _{r \rightarrow \tilde{r}+} z(r)=+\infty,
$$

which would prove the lemma by contradicting the maximality of $I$.

So assume that $\tilde{r}>r_{1}$ and $A(\tilde{r})=0$. To verify (3.3), note that since $\lim _{r \rightarrow \tilde{r}} A(r)=0$, it follows that for $r$ sufficiently close to $\tilde{r}$, we can find a constant $C_{0}>0$ such that

$$
C \equiv \frac{(1+\mu)(1+3 \mu z)}{2 \sigma}-\frac{3(1-z) A}{1-A} \geqq C_{0} .
$$


Thus for $r$ sufficiently close to $\tilde{r}$, it follows that

$$
z^{\prime}=-C \frac{z}{r} \frac{1-A}{A}<0
$$

in view of (2.19) and the fact that $z>0$ on $J$ by Lemma 2. Thus for $r$ sufficiently close to $\tilde{r}, z(r) \geqq \delta$ for some $\delta>0$. Hence, there exists an interval $\left(\tilde{r}, \tilde{r}_{0}\right]$, in which we have

$$
\begin{gathered}
z^{\prime}(r) \leqq-\frac{C_{1}}{A}, \\
A^{\prime}(r) \leqq C_{2},
\end{gathered}
$$

for some positive constants $C_{1}$ and $C_{2}$. Thus integrating (3.5) gives

$$
A(r) \leqq C_{2}(r-\tilde{r})
$$

for all $r \in\left(\tilde{r}, \tilde{r}_{0}\right]$. Substituting this into (3.4) gives

$$
z^{\prime}(r) \leqq-\frac{C_{1}}{C_{2}(r-\tilde{r})}
$$

Now let $s \in\left(\tilde{r}, \tilde{r}_{0}\right)$. Then integrating (3.7) from $s$ to $\tilde{r}_{0}$ gives

$$
z\left(\tilde{r}_{0}\right)-z(s) \leqq-\frac{C_{1}}{C_{2}} \ln \frac{\tilde{r}_{0}-\tilde{r}}{s-\tilde{r}},
$$

so that

$$
z(s) \geqq z\left(\tilde{r}_{0}\right)+\frac{C_{1}}{C_{2}} \ln \frac{\tilde{r}_{0}-\tilde{r}}{s-\tilde{r}} .
$$

Letting $s \rightarrow \tilde{r}$, in (3.9) gives the conclusion (3.3). This completes the proof of Lemma 3.

Note that in the case $r_{1}>0$, if $z$ is uniformly bounded in a neighborhood $r_{1}<r<r_{1}+\delta$ of $r_{1}$, then from (2.20), the derivative $A^{\prime}(r)$ is uniformly bounded on this neighborhood, and thus $A$ has a value and is continuous from the right at $r_{1}$. Thus the proof of the above lemma directly implies

Corollary 2. If $r_{1} \geqq 0$ and if $\lim _{r_{n} \rightarrow r_{1}+} A\left(r_{n}\right)=0$ for some sequence $r_{n} \rightarrow r_{1}+$, then $\lim _{r \rightarrow r_{1}}+z(r)=+\infty$.

We now have that $0<A<1$ on the maximal interval $I$, and this will be used to prove

Lemma 4. Suppose $z(\tilde{r}) \geqq 1$ for some $\tilde{r} \in I$. Then $z^{\prime}(r)<0$ and $A^{\prime}(r)<0$ for all $r \in\left(r_{1}, \tilde{r}\right)$. (This applies when $r_{1}>0$ and when $r_{1}=0$.)

Proof. Lemmas 1-3 imply that $0<A<1$ and $z>0$ throughout the interval $I$, and so by (2.21), $C>0$ at $r=\tilde{r}$. Thus (2.19) and (2.20) imply that $z^{\prime}(\tilde{r})<0$ and $A^{\prime}(\tilde{r})<0$, and this is clearly maintained for $r<\tilde{r}$.

The next lemma demonstrates that when $r_{1}>0, z$ tends to infinity as $r$ tends to $r_{1}+$. 
Lemma 5. Let $r_{1}>0$; then $\lim _{r \rightarrow r_{1}} z(r)=+\infty$.

Proof. First, if $A$ tends to zero on some subsequence $r_{n} \rightarrow r_{1}+$, then Corollary 2 implies that $\lim _{r \rightarrow r_{1}+z}(r)=+\infty$. Thus we may assume that there is some $\delta>0$ such that $\delta<A(r)<1$ for all $r \in\left(r_{1}, r_{0}\right.$ ] (in light of the fact that $0<A<1$ away from $\left.r_{1}\right)$. In this case, we see from (2.19)-(2.21) that if $z$ is uniformly bounded on $I$, then $C(1-A)$ is uniformly bounded on $I$, and hence that $z, A, z^{\prime}$, and $A^{\prime}$ all have finite limits at $r_{1}$. This contradicts the maximality of $I$. Thus there must be a sequence $r_{k}$ tending to $r_{1}$ from above, such that $z\left(r_{k}\right)$ tends to $+\infty$, and so by the last lemma, $\lim _{r \rightarrow r_{1}+} z(r)=+\infty$. This completes the proof of the lemma.

The next lemma shows that $M(r)$ tends to 0 at $r_{1}$.

Lemma 6. The mass function $M(r)$ satisfies

$$
\lim _{r \rightarrow r_{1}+} M(r)=0 .
$$

Proof. First note that $M^{\prime}(r)>0$ for all $r \in\left(r_{1}, r_{0}\right]$ because $M^{\prime}(r)=4 \pi \rho r^{2}$ and $\rho>0$ (in view of (2.1), (2.2), our initial conditions, $A>0$ and the fact that we assume (2.11), so that $\left.\rho^{\prime} \equiv \frac{p^{\prime}}{\sigma}>0\right)$.

Consider first the case $r_{1}=0$. Writing

$$
\frac{2 \mathscr{G} M}{r}=1-A
$$

we see that if $M \rightarrow M_{0} \neq 0$ as $r \rightarrow 0$, then $A \rightarrow \pm \infty$ as $r \rightarrow 0+$, which violates the fact that $0<A<1$ on $I$. Thus $M_{0}=0$ when $r_{1}=0$.

Consider next the case $r_{1}>0$. In view of (3.10), in order to show that $M(r) \rightarrow 0$ as $r \rightarrow r_{1}+$, it suffices to show that

$$
\lim _{r \rightarrow r_{1}+} A(r)=1 .
$$

To show this, assume for contradiction that

$$
\lim _{r \rightarrow r_{1}+} A(r) \neq 1 .
$$

Now consider the identity (3.1) which we can write as

$$
\frac{1-A(r)}{1-A\left(r_{0}\right)}=\exp \left[-\int_{r_{0}}^{r}(1-3 z) \frac{d r}{r}\right] .
$$

Since we know already that $z$ tends to $+\infty$ at $r_{1}$, it follows from (3.13) that $A$ tends to 1 at $r_{1}$ if $z(r)$ has a non-integrable singularity at $r=r_{1}$, thereby violating (3.12).

We now show that $z(r)$ has a non-integrable singularity at $r=r_{1}$. To do this, note first that $A(r)$ has a finite limit at $r=r_{1}$ because $z>1$ near $r=r_{1}$, so from (2.20), $A^{\prime}<0$, and $A<1$ on $I$. Thus by (3.12), there exists a $\delta>0$ such that

$$
\delta<A(r)<1-\delta
$$


throughout the interval $I$, and so from (2.19), there exists a constant $C_{1}>0$ such that for $r$ near $r_{1}$, say $r \in\left(r_{1}, r_{2}\right)$,

$$
z^{\prime} \leqq-C_{1} z^{2}
$$

We show that $z(r)$ is not integrable at $r=r_{1}$ as follows: Let $\tilde{z}(r)$ be the solution of

$$
\begin{gathered}
\tilde{z}^{\prime}=-C_{1} \tilde{z}^{2}, \\
\tilde{z}\left(r_{2}\right)=\tilde{z}_{2} .
\end{gathered}
$$

Integrating (3.15) gives

$$
\tilde{z}(r)=\frac{1}{C_{1}\left[r-\left(r_{2}-\frac{1}{C_{1} \tilde{z}_{2}}\right)\right]} .
$$

Now choose $\tilde{z}_{2}$ so that

$$
r_{2}-\frac{1}{C_{1} \tilde{z}_{2}}=r_{1}
$$

that is,

$$
\tilde{z}_{2}=\frac{1}{C_{1}\left(r_{2}-r_{1}\right)} .
$$

Thus $\tilde{z}(r)$ is defined throughout the interval $\left(r_{1}, r_{2}\right]$, and by (3.17),

$$
\tilde{z}(r)=\frac{1}{C_{1}\left(r-r_{1}\right)},
$$

so that $\tilde{z}(r)$ has a non-integrable blowup precisely at $r=r_{1}$. We now compare $\tilde{z}(r)$ to $z(r)$. To this end, choose $\tilde{C}$ such that

$$
z(r) \geqq \tilde{z}(r)+\tilde{C}
$$

for all $r \in\left(r_{1}, r_{2}\right]$. Note that we can achieve (3.19) because $z^{\prime}(r) \leqq \tilde{z}^{\prime}(r)$ for all $r \in\left(r_{1}, r_{2}\right]$, and so (3.19) holds if

$$
z\left(r_{2}\right)<\tilde{z}\left(r_{2}\right)+\tilde{C}
$$

Thus to achieve (3.19), we need only choose $\tilde{C}>z\left(r_{2}\right)-\tilde{z}\left(r_{2}\right)$. Since $z^{\prime}<\tilde{z}^{\prime}$, (3.19) holds for all $r \in\left(r_{1}, r_{2}\right]$. Hence, in light of (3.18), (3.19) implies that $z(r)$ is non-integrable at $r=r_{1}$. Therefore, (3.13) implies that $A \rightarrow 1$ and $M \rightarrow 0$ as $r \rightarrow r_{1}+$, and this contradicts equation (3.12) and completes the proof of the lemma.

An argument similar to (3.14)-(3.19) shows that if $r_{1}>0$, then $\rho$ and $p$ have

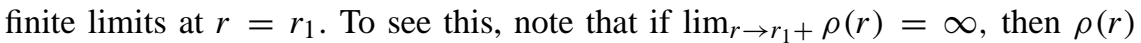
would have a non-integrable singularity at $r=r_{1}$ because the right-hand side of the $\mathrm{O}-\mathrm{V}$ equation (2.1) is quadratic in $\rho$. This would imply that

$$
\lim _{r \rightarrow r_{1}+} M(r)=M\left(r_{0}\right)-\lim _{r \rightarrow r_{1}+} \int_{r}^{r_{0}} 4 \pi \rho(s) s^{2} d s=-\infty,
$$


thereby contradicting Lemma 6. Thus $\rho(r)$ and $p(r)=\frac{\rho(r)}{\sigma(r)}$ must have finite values at $r=r_{1}$ when $r_{1}>0$. This, together with $p^{\prime}<0$, (by the O-V equation), and $\rho^{\prime}<0$, (since $\sigma>0$ ), implies (2.33) and (2.34). To verify (2.35), note that if $r_{1}>0$, then since we assume that $p^{\prime}<0$, the $\mathrm{O}-\mathrm{V}$ equation implies that $\rho^{\prime}<0$, and we must have $\rho>0$ for $r<r_{1}$. Thus $M^{\prime}(r)=4 \pi \rho(r) r^{2}>0$. Thus either $p$ tends to infinity before $r=0$, or else $M(r)$ tends to $M(0)<0$ as $r \rightarrow 0$. But the latter contradicts $p^{\prime}<0$ because (2.16) implies that $p^{\prime}<0$ for sufficiently small $r$ when $\rho$ is bounded.

We next show that (2.32) and (2.30) hold for the case $r_{1}=0$. For this, first note that (2.32) follows because $M(0)=0$ and $\rho^{\prime}<0$. Indeed,

$$
\begin{aligned}
\bar{\rho}(r)=\frac{3}{4 \pi} \frac{M(r)}{r^{3}} & =\frac{3}{4 \pi} \frac{1}{r^{3}} \int_{0}^{r} 4 \pi \rho(s) s^{2} d s \\
& \geqq \frac{3}{4 \pi} \frac{\rho(r)}{r^{3}} \int_{0}^{r} 4 \pi s^{2} d s=\rho(r)
\end{aligned}
$$

hence $z(r) \leqq 1$ for all $r \in I$, and (2.32) is established. For (2.30), note that if $\rho(r) \rightarrow \rho(0)<\infty$ as $r \rightarrow 0$, then we can write $\rho(r)=\rho(0)+o(r)$. This implies that for $r$ near zero,

$$
M(r)=\int_{0}^{r} 4 \pi \rho(s) s^{2} d s=\frac{4 \pi}{3} \rho(0) r^{3}+o\left(r^{3}\right) .
$$

Thus

$$
\bar{\rho} \equiv \frac{3}{4 \pi} \frac{M(r)}{r^{3}}=\rho(0)+o(r),
$$

and so

$$
z(r)=\frac{\rho(r)}{\bar{\rho}(r)} \rightarrow 1,
$$

as $r \rightarrow 0$. Moreover, by L'Hôpital's rule,

$$
\lim _{r \rightarrow 0} \frac{M(r)}{r}=\lim _{r \rightarrow 0} M^{\prime}(r)=\lim _{r \rightarrow 0} 4 \pi \rho(r) r^{2}=0,
$$

so that $(2.30)$ holds.

To verify (2.26), first note that from (2.22) we have

$$
(\ln B)^{\prime}=\frac{(1+3 \tilde{\mu} z)(1-A)}{r A},
$$

so that if $B \rightarrow 0$ for some $r \rightarrow \tilde{r} \in\left(r_{1}, r_{0}\right)$, then $\ln B \rightarrow-\infty$ as $r \rightarrow \tilde{r}$. But by (3.20), we see that $(\ln B)^{\prime}$ is bounded near $\tilde{r}$, and this is a contradiction; thus (2.26) holds. Moreover, when $r_{1}>0,(2.30)$ holds because $M\left(r_{1}\right)=0$, and for (2.31), integrate (3.20) from $r$ to $\tilde{r}, r_{1}<r<\tilde{r}$, to obtain

$$
B(r)=B(\tilde{r}) \exp \left[-\int_{r}^{\tilde{r}} \frac{(1+3 \mu z)}{s} \frac{1-A}{A} d s\right] .
$$


But $z$ is of order $(1-A)^{-1}$ as $r \rightarrow r_{1}$ and $A \rightarrow 1$, so the integrand is bounded, and hence $B\left(r_{1}\right)>0$. To show that (2.31) holds when $r_{1}=0$ and $\rho$ has a finite limit at $r=0$, note that in this case $z$ tends to unity as $r \rightarrow 0$ and $\frac{1-A}{r}=\frac{2 \mathscr{G} M}{r^{2}}$ has a finite limit at $r=0$ by L'Hôpital's Rule, so (3.21) implies that $B(r)$ has a finite positive limit as $r \rightarrow 0$ in this case as well.

To complete the proof of Theorem 1, it remains only to verify (2.35). So assume that $r_{1}>0$, and $p^{\prime}<0$ for $r<r_{1}$. In this case, $\sigma>0$ implies $\rho^{\prime}<0$, and so also $M^{\prime}>0$ and $A>1$ on the smooth continuation of the O-V system (2.1), (2.2) to a maximal interval of existence $J \equiv\left(r_{2}, r_{1}\right], 0 \leqq r_{2}<r_{1}$. Since $\rho^{\prime}>0$, it follows directly from the maximality of $J$ that either $\rho \rightarrow+\infty$ as $r \rightarrow r_{2}+$, with $r_{2}>0$, in which case we are done, or else $r_{2}=0$. But if $r_{2}=0$ and $\rho$ (hence $p$ and $M$ ) have finite limits at $r=0$, then near $r=0, A \sim-2 M(0) / r$, and thus it follows from (2.1) that the equation for $\rho$ can be estimated above and below by an equation of the form

$$
\rho^{\prime}=-D \frac{\rho}{r}
$$

for some postitive constant $D$. But solutions of (3.22) take the form

$$
\rho(r)=\rho\left(r_{1}\right)\left(\frac{r_{1}}{r}\right)^{D}, \quad 0<r<r_{1},
$$

and so $\rho$ must blow up at $r=0$. This contradicts the boundedness of $\rho$, and hence establishes (2.35). This completes the proof of Theorem 1.

As a final comment note that Theorem 1 implies that black-holes cannot form at any positive radius in smooth solutions of the $\mathrm{O}-\mathrm{V}$ system starting from initial conditions (2.23). Indeed, we have shown that $A>0$ on the interval $I$, so when $r_{1}=0, A$ cannot be zero at any positive value of $r$. Thus, the only case to consider is the case when $r_{1}>0$. In this case, since $M^{\prime}>0$, we must have that $M<0$ throughout the smooth continuation of the O-V system (2.1), (2.2) to values of $0<r<r_{1}$. Thus $A>1$ on this extended domain.

Acknowledgement. The work of SMOLLER was supported in part by NSF Applied Mathematics Grant Number DMS-95OOO-694, in part by ONR, US NAVY grant number N00014-94-1-0691, and by the Institute of Theoretical Dynamics (ITD), UC-Davis. J. SMOLLER thanks JoEL KEIZER, director of the ITD, for his warm hospitality during SMOLLER's tenure as a Visiting Regents Professor at UC-Davis. The work of TEMPLE was supported in part by NSF Applied Mathematics Grant Number DMS-95OOO-694, in part by ONR, US NAVY grant number N00014-94-1-0691, a Guggenheim Fellowship, and by the Institute of Theoretical Dynamics, UC-Davis.

\section{References}

1. S. Weinberg, Gravitation and Cosmology: Principles and Applications of the General Theory of Relativity, John Wiley \& Sons, New York, 1972.

2. R. Courant \& K.O. Friedrichs, Supersonic Flow and Shock Waves, John Wiley \& Sons, New York, 1948.

3. J. Smoller \& B. Temple, Shock-wave solutions of the Einstein equations, Arch. Rational Mech. Anal., 128 (1994), 249-297. 
4. J. Smoller \& B. Temple, Astrophysical shock-wave solutions of the Einstein equations, Phys. Rev. D, 51 (1995), 2733-2743.

5. J. Smoller \& B. Temple, Solutions of the Oppenheimer-Volkoff equations inside 9/8'ths of the Schwarzschild radius, Comm. Math. Phys., 184 (1997), 597-617.

Department of Mathematics

University of Michigan

Ann Arbor, Michigan 48109

and

Department of Mathematics

University of California

Davis, California 95616 